\title{
The role of promastigote secretory gel in the origin and transmission of the infective stage of Leishmania mexicana by the sandfly Lutzomyia longipalpis
}

\author{
M. E. ROGERS*, M. L. CHANCE and P. A. BATES \\ Division of Molecular Biology and Immunology, Liverpool School of Tropical Medicine, Pembroke Place, \\ Liverpool L3 $5 Q A, U K$
}

(Received 13 Fune 2001; revised 3 December 2001; accepted 3 December 2001)

\begin{abstract}
SUMMARY
Transmission of leishmaniasis is effected by a specific developmental stage, the metacyclic promastigote. The precursors of metacyclic promastigotes were a distinct subpopulation of parasites, identified for the first time as a new stage in the life-cycle and named leptomonad promastigotes. Microdissection of infected sandflies into 4 midgut regions and foregut allowed precursor-product relationships to be established for amastigote-procyclic promastigote, procyclic-nectomonad promastigote, nectomonad-leptomonad promastigote and leptomonad-metacyclic promastigote developmental switches. Metacyclic promastigotes occurred mainly in the thoracic midgut and cardia, coincident with the accumulation of a promastigote secretory gel (PSG) plug in these anterior regions. The gel-like plug was isolated from flies with mature infections and found to contain predominantly leptomonad promastigotes. The PSG plug also contained the majority $(75 \%)$ of the total metacyclic promastigote population in the sandflies, which were concentrated at the anterior pole. The PSG plug was found to be the main site of metacyclogenesis, and acted as a reservoir of leptomonad promastigotes from which metacyclic forms differentiated and migrated forward to promote the infective potential of the fly. The PSG plug occluded and distorted the midgut, forcing the stomodeal valve open and affecting the feeding success of the sandflies, such that they experienced difficulty in taking a full meal. Collectively, these data support the role of the PSG in the transmission of leishmaniasis, by conditioning the midgut environment for metacyclogenesis and altering the feeding ability of infected sandflies.
\end{abstract}

Key words: Leishmania, sandfly, metacyclic promastigote, promastigote secretory gel (PSG), transmission.

\section{INTRODUCTION}

Parasites of the genus Leishmania are protozoa that occur within the phagolysosomes of the mammalian macrophages as non-flagellated amastigotes, and as motile flagellated promastigotes in phlebotomine sandflies. Infection of the sandfly begins after taking a bloodmeal from an infected host. Ingested amastigotes transform within the digesting bloodmeal and undergo development as a number of promastigote morphological forms (Killick-Kendrick, 1979). Upon engorgement the bloodmeal is encased by a peritrophic matrix, a chitinous sac formed from secretions of the midgut epithelium. Therein amastigotes transform into ovoid procyclic and long slender nectomonad forms, which initiate the growth of the infection. Towards the end of digestion of the bloodmeal the parasites escape from the peritrophic matrix to avoid expulsion during defecation, and further resist this process by attaching to the midgut microvillar border (Pimenta et al. 1992; Sacks et al. 1994). Subsequently, colonization of the anterior

* Corresponding author: Tel: +44 151708 9393. Fax: +44 151708 9007. E-mail: m.e.rogers@liverpool.ac.uk regions of the midgut is accompanied by transformation to a variety of morphological forms, which include broad attached haptomonad promastigotes, attached and free-swimming paramastigotes and small, highly motile and non-dividing metacyclic promastigotes (Killick-Kendrick, 1990; Walters 1993; Lawyer et al. 1990 and references therein). The precise temporal and spatial relationships between these various promastigote forms and how they produce transmissible infections remain unclear.

Successful Leishmania development in sandflies results in the generation and transmission of the mammal-infective form, the metacyclic promastigote (Sacks, 1989; Saraiva et al. 1995). Surprisingly, little is known of the direct precursors of metacyclic promastigotes or the conditions that influence metacyclogenesis in vivo. A commonly observed feature of the Leishmania/sandfly combinations examined so far is the presence of a gel-like material that blocks the lumen of the anterior midgut (Walters et al. 1989a; Lawyer et al. 1990). Recently, promastigotes of L. mexicana and L. major were demonstrated to secrete this proteophosphoglycanrich promastigote secretory gel (PSG) in sandflies 
(Stierhof et al. 1999). These observations have fuelled the idea that the blocked state of the infected sandfly may frustrate normal blood feeding and, therefore, promote transmission by causing more frequent biting and/or for longer feeding. Furthermore, it has been proposed that infected sandflies transmit leishmaniasis by regurgitation of metacyclic promastigotes when the blockage is finally cleared (Shortt \& Swaminath, 1928; Killick-Kendrick, 1979). In the present study, the spatial variation of the parasites embedded within the PSG is examined for the first time. We propose a role for this subpopulation of parasites, which we name leptomonad promastigotes, in generating and maintaining the population of mammal-infective metacyclic promastigotes. In addition, evidence is given that the accumulation of PSG promotes transmission by altering the feeding success of heavily infected sandflies and conditioning the midgut in favour of metacyclogenesis.

\section{MATERIALS AND METHODS}

\section{Maintenance and infection of sandfies}

A laboratory colony of Lutzomyia longipalpis established from flies caught in Jacobina, Bahia, Brazil (Jacobina strain) was used in all experiments, and maintained using standard methods (Modi, 1997). Five-day-old female flies were infected by feeding through a chick skin on whole rabbit blood seeded with lesion amastigotes of Leishmania mexicana (MNYC/BZ/62/M379) at $2 \times 10^{6} / \mathrm{ml}$ (Bates, 1997). Amastigotes were obtained from rump lesions of $\mathrm{BALB} / \mathrm{c}$ mice by dissection and homogenization in culture medium: Medium 199 supplemented with $10 \%(\mathrm{v} / \mathrm{v})$ heat-inactivated foetal calf serum, BME vitamins (all from Gibco), $25 \mu \mathrm{g}$ gentamicin sulphate/ml (Sigma), pH 7·2; and used immediately. Subsequent to blood-feeding, female flies were maintained at $25-27{ }^{\circ} \mathrm{C}, 80-95 \%$ relative humidity and supplied with $70 \%(\mathrm{w} / \mathrm{v})$ sucrose ad libitum. This experimental parasite-vector combination has been previously shown to support full development of the parasite, including acquisition and transmission of infections by bite (Ismaeel et al. 1998).

\section{Dissection of infected sandflies}

For each sandfly, first the head was severed at the junction with the thorax and retained for analysis of foregut populations. The remainder of the gut was removed by dissection from the posterior end of the abdomen. Only guts that were removed without damage were used in further analysis. In some experiments the gut was further subdivided into 4 regions: cardia, thoracic midgut, anterior abdominal midgut, and posterior abdominal midgut. The number of promastigotes was determined by homogenizing intact dissected midguts or gut regions in $30 \mu \mathrm{l}$ of culture medium in $1.5 \mathrm{ml}$ microfuge tubes using a Teflon-coated pestle. Parasites were counted in an improved Neubauer haemocytometer after fixation in $2 \%(\mathrm{w} / \mathrm{v})$ formaldehyde in phosphatebuffered saline. To isolate the PSG plugs containing parasites (found in the anterior midgut of mature infections), guts were carefully sliced open in a longitudinal direction, and each plug lifted out with mounted entomological needles. In some experiments PSG plugs were subdivided into 3 equal sections, anterior pole, mid-section and posterior pole. Histological sections of infected sandflies were prepared as described previously (Ismaeel et al. 1998).

\section{Morphological analysis of parasites}

The morphology of the parasites was analysed using bright-field light microscopy. Ten $\mu$ l volumes of infected gut homogenate or egested parasites were placed on microscope slides, air dried, fixed in methanol and stained with $10 \%(\mathrm{v} / \mathrm{v})$ Giemsa's stain. Observations and measurements were made under oil immersion at $1000 \times$ magnification using an eyepiece graticule. Parasites were placed into 1 of 7 categories (Table 1), based on previous descriptions (Killick-Kendrick, Molyneux \& Ashford, 1974; Molyneux \& Killick-Kendrick, 1987) and the evidence of this study. Six of these are: amastigote, the form of Leishmania found in the mammalian host; procyclic promastigotes, the first promastigote form that develops in the sandfly; nectomonad promastigotes, an elongated promastigote form; haptomonad promastigotes, a form with an expanded flagellar tip that is used to attach to cuticular surfaces in vivo; metacyclic promastigotes, the mammalinfective form; and paramastigotes, a flagellated form in which the kinetoplast is adjacent to the nucleus. A new category is defined in the current report: the leptomonad promastigote (redefined after Wenyon, 1926). Evidence that leptomonad promastigotes are a functionally distinct form with a role in generation of the PSG plug and precursor to metacyclic promastigotes is provided in this study. Any forms observed that did not fall into these 7 main categories, were placed into 'others'.

\section{Determination of haemoglobin content of sandfly homogenate}

A colorometric method adapted from Briegel, Lea \& Klowden (1979) was used. After brief centrifugation (1800 $\boldsymbol{g}$ for $30 \mathrm{sec}$ ) to remove large debris, $20 \mu \mathrm{l}$ of sandfly gut homogenate was mixed with $200 \mu \mathrm{l}$ of Drabkin's reagent (Sigma) in the dark for $30 \mathrm{~min}$. Then $200 \mu$ l samples were transferred to a microtitre 

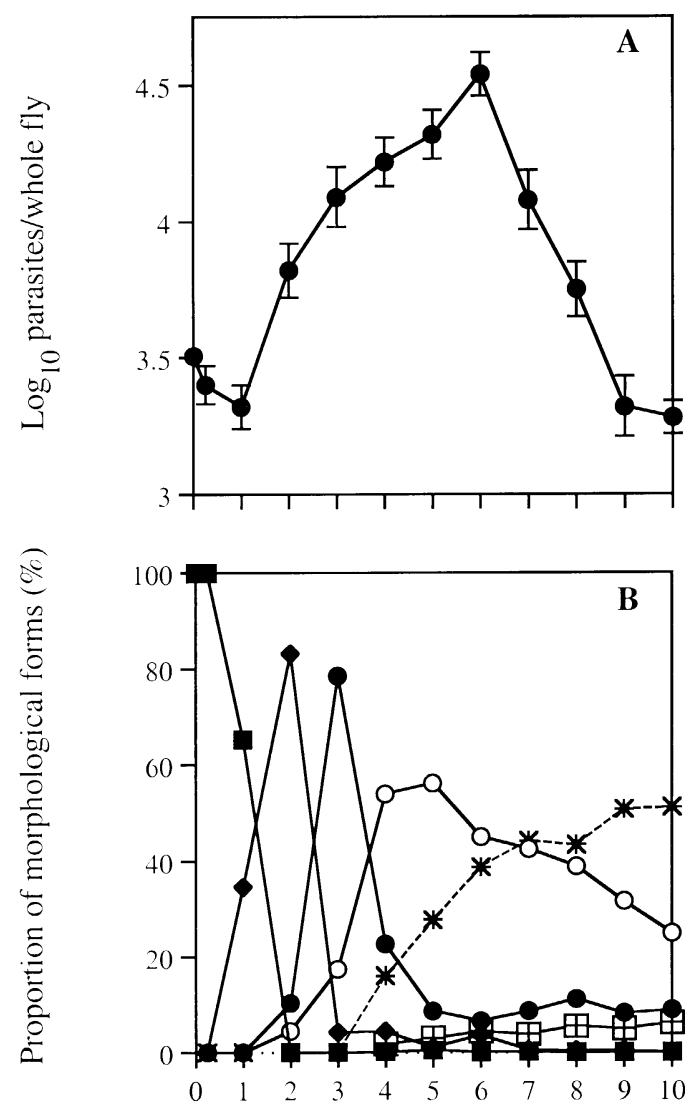

Days post-infection

Fig. 1. General development of Leishmania mexicana in Lutzomyia longipalpis. (A) Growth of the parasite population. Flies were infected with $2 \times 10^{6}$ amastigotes/ $\mathrm{ml}$ suspended in rabbit blood and the total numbers of parasites per fly determined over a 10 -day period. The data represent the geometric mean of flies analysed from 2 independent experiments, each point representing 20 infected flies (10 from each experiment). The bars indicate 1 standard error from the mean. (B) Developmental profile. Samples were prepared for morphological analysis and a minimum of 200 parasites were analysed per fly. The relative proportions of amastigotes $(\boldsymbol{\square})$, procyclic promastigotes $(\bullet)$, nectomonad promastigotes $(\mathbf{)})$, leptomonad promastigotes $(\bigcirc)$, metacyclic promastigotes (*) and haptomonad promastigotes $(\boxplus)$ are shown (see Table 1 for definitions). Each point represents the arithmetic mean of 20 infected flies from 2 independent experiments. The dataset represents the analysis of approximately 44000 individual parasites.

plate and assayed spectrophotometrically at $540 \mathrm{~nm}$ after blanking on the same volume of Drabkin's reagent. Whole rabbit blood was used for calibration.

\section{Feeding activity of infected sandflies}

Infected sandflies were allowed to feed either on a chick skin membrane (as described above) or on groups of 5 female BALB/c mice. In the latter, each group of mice was anaesthetized and placed into a netting cage with approximately 50 sandflies with 7day-old infections. Access to the head was prevented, by placing inside a small hood, and the base of the tail was screened with tape, allowing flies free access to the other parts of the body. Mice were exposed for $30 \mathrm{~min}$, then removed from the cage. Flies fed exclusively on the exposed skin of the limbs. After feeding, flies were dissected, their feeding status recorded (none, partial or full meal taken) and the infection level in the gut assessed by haemocytometer counting or light microscopy. In the latter the infection level was recorded as: uninfected, no parasites observed; light infection, less than 1000 parasites observed; or heavy infection, over 1000 parasites observed. Mice were allowed to recover from anaesthesia and the development of lesions monitored weekly.

\section{RESULTS}

\section{Development of Leishmania mexicana in sandflies}

To determine the identity of the developmental precursors of the metacyclic promastigotes in $L$. mexicana-infected sandflies a quantitative analysis of development was performed (Fig. 1).

In fresh bloodmeals the initial number of parasites in infected flies was estimated both by haemocytometer counting and from haemoglobin analysis of the fresh bloodmeal, calibrated against whole rabbit blood. This yielded a value of $1.6 \mu \mathrm{l}$ of blood ingested during feeding, and indicated that an average of 3200 amastigotes had been ingested by individual flies. Note that the volume of blood ingested is greater than the volume of the bloodmeal in the gut, since the latter is concentrated by prediuresis, resulting in a gel-like mixture of erythrocytes and serum proteins (Sádlová, Reishig \& Volf, 1999). Measurement of the dimensions of the bloodmeal in recently fed flies (1-2 $\mathrm{h}$ post-feeding) gave a volume of approximately $1 \mu$ l. Interestingly, the numbers of parasites per fly recorded at $6 \mathrm{~h}$ (day 0 ) and $24 \mathrm{~h}$ (day 1) were lower than the initial density, $80 \%$ and $66 \%$, respectively, indicating that a proportion of parasites were killed early in the infection. Thereafter, the number of parasites per fly steadily increased to a peak on day 6 (approximately 35000 per fly in these infections), before decreasing again to a level similar to the initial number by day 10. The peak number per fly recorded on day 6 was approximately 16 times the number recorded at the lowest point on day 1 (Fig. 1A).

In addition to determining the number of parasites present in infected sandflies, the proportions of different morphological forms were determined by examining Giemsa-stained preparations (Fig. 1B). Amastigotes were the only form detected up to $6 \mathrm{~h}$ post-feeding, indicating that it was parasites under- 
Table 1. Morphological categories of Leishmania mexicana

(Parasites were examined by bright field microscopy and placed into 1 of the 7 categories defined above. Any parasites not falling within these categories were placed into "others". Illustrations of different forms are all displayed at the same magnification.)

\begin{tabular}{|c|c|c|}
\hline Morphological category & Criteria & Illustration \\
\hline Amastigote & $\begin{array}{l}\text { Ovoid body form, no flagellum } \\
\text { protruding from flagellar pocket }\end{array}$ & \\
\hline Procyclic promastigote & $\begin{array}{l}\text { Body length } 6 \cdot 5-11.5 \mu \mathrm{m} \text {, flagellum }<\text { body } \\
\text { length (body width variable) }\end{array}$ & \\
\hline Nectomonad promastigote & $\begin{array}{l}\text { Body length } \geq 12 \mu \mathrm{m} \text {, (body width and } \\
\text { flagellar length variable) }\end{array}$ & \\
\hline Leptomonad promastigote & $\begin{array}{l}\text { Body length } 6 \cdot 5-11 \cdot 5 \mu \mathrm{m} \text {, flagellum } \geq \text { body } \\
\text { length (body width variable) }\end{array}$ & \\
\hline Haptomonad promastigote & $\begin{array}{l}\text { Disc-like expansion of flagellar tip } \\
\text { (body form and flagellar length variable) }\end{array}$ & \\
\hline Metacyclic promastigote & $\begin{array}{l}\text { Body length } \leq 8 \mu \mathrm{m} \text {, body width } \leq 1 \cdot 0 \mu \mathrm{m} \text {, } \\
\text { flagellum }>\text { body length }\end{array}$ & \\
\hline Paramastigote & $\begin{array}{l}\text { Kinetoplast adjacent tonucleus, } \\
\text { external flagellum present }\end{array}$ & \\
\hline
\end{tabular}

going transformation to procyclic promastigotes (Table 1) that were reduced in numbers at an early point in the infection. By $24 \mathrm{~h}$ (day 1) $35 \%$ of the remaining amastigotes had transformed into procyclic promastigotes, and by day 2 amastigotes had disappeared completely, being replaced by a population predominating in procyclic forms $(83 \%)$. The appearance of this first peak composed of procyclic promastigotes $(6000 / \mathrm{fly})$ was co-incident with an increase in the number of parasites per fly (Fig. 1A), indicating that transformation was followed by the onset of replication. Two other forms were observed at low prevalence for the first time in day 2 samples: nectomonad promastigotes $(10 \%)$ and leptomonad promastigotes $(5 \%)$. Both of these forms continued to increase in proportion, particularly nectomonad promastigotes, which became the predominant form $(79 \%$ ) and second major peak by day 3 (10000/fly), with the procyclic promastigotes rapidly falling to less than $5 \%$ of the total. Nectomonad promastigotes decreased rapidly, falling back to approximately $10 \%$ by day 5 , and persisted at this low level for the duration of the infections. Leptomonad promastigotes increased in proportion more slowly, rising to approximately 54 and $56 \%$ of the population on days 4 and 5, respectively, and constituting the third major peak (17000/fly). Two forms made their first appearance on day 4: haptomonad promastigotes
$(2 \%)$ and metacyclic promastigotes $(16 \%)$. Haptomonad forms were polymorphic, a minor population was seen with an extended flagellum, but most possessed a very short flagellum and expanded tip (Table 1). The cell bodies were usually tear- or pearshaped. Haptomonad promastigotes persisted at low prevalence for the duration of the infections. Metacyclic promastigotes were readily distinguished in fresh preparations by their small cell bodies, long flagella and vigorous motility. Metacyclic promastigotes gradually increased in proportion from day 4 onwards, so that by day 10 they represented $51 \%$ and the fourth peak of the population. These increases in proportion were made at the expense of the leptomonad forms, which steadily decreased from their peak on days $4-5(55 \%)$ to $25 \%$ of the total by day 10 . Paramastigotes were rarely seen, never accounting for more than $2 \%$ of the total, and 'others' (not falling into any of the 7 main categories) represented less than $1 \%$ of the total parasites observed. These data are clear evidence for precursor-product relationships between procyclicnectomonad promastigote, nectomonad-leptomonad promastigote and leptomonad-metacyclic promastigote.

The purpose of a female sandfly in taking a bloodmeal is to obtain nutrients for egg production, and the development of Leishmania takes place 

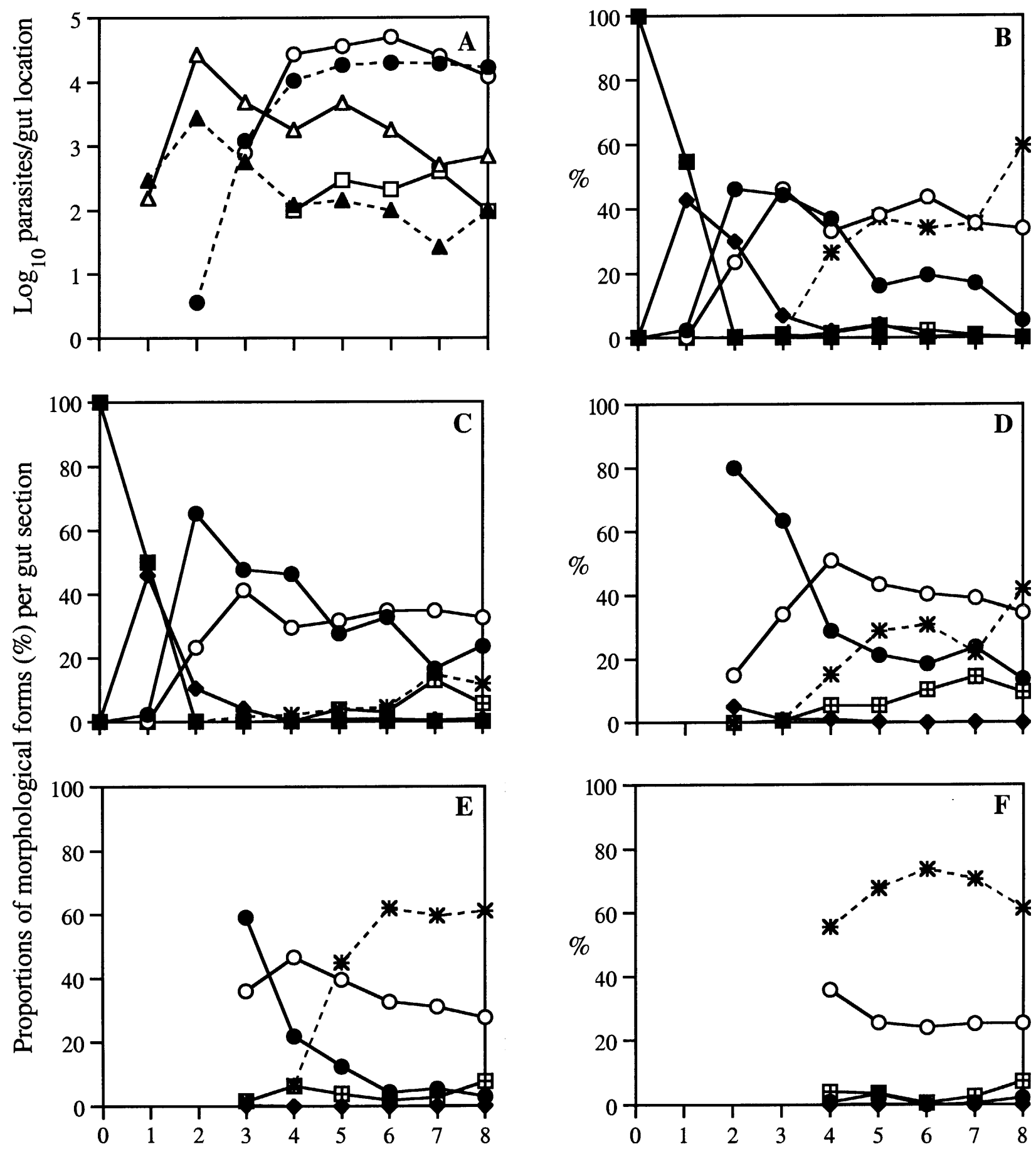

Fig. 2. Development of Leishmania mexicana within different gut regions of Lutzomyia longipalpis. (A) Growth in different gut regions. Flies were fed by membrane feeding on rabbit blood seeded with $2 \times 10^{6}$ amastigotes $/ \mathrm{ml}$. On each day for 8 days, 10 or more infected flies were dissected and the gut of each subdivided into 5 regions (see Fig. 4 for details). The number of parasites in each region was determined. Each point represents the geometric mean of at least 10 infected flies from 2 independent experiments. The gut regions were: foregut $(\square)$, cardia $(\bigcirc)$, thoracic midgut $(\mathbf{O})$, anterior abdominal midgut $(\triangle)$, and posterior abdominal midgut $(\mathbf{\Delta})$. (B-F) Developmental profile within different gut regions. On each day samples were also prepared for morphological analysis. A minimum of 200 parasites were examined per region per fly, and the relative proportions of amastigotes ( $\mathbf{\square}$ ), procyclic promastigotes $(\diamond)$, nectomonad promastigotes $(\bigcirc)$, leptomonad promastigotes $(\bigcirc)$, metacyclic promastigotes $(*)$ and haptomonad promastigotes $(\boxplus)$ determined. The diagram shows data from: $(\mathrm{B})$ the posterior abdominal midgut; (C) anterior abdominal midgut; (D) thoracic midgut; (E) cardia and (F) foregut. Each point represents the arithmetic mean of 10 or more infected flies from 2 independent experiments. The dataset represents the analysis of approximately 52000 individual parasites. 


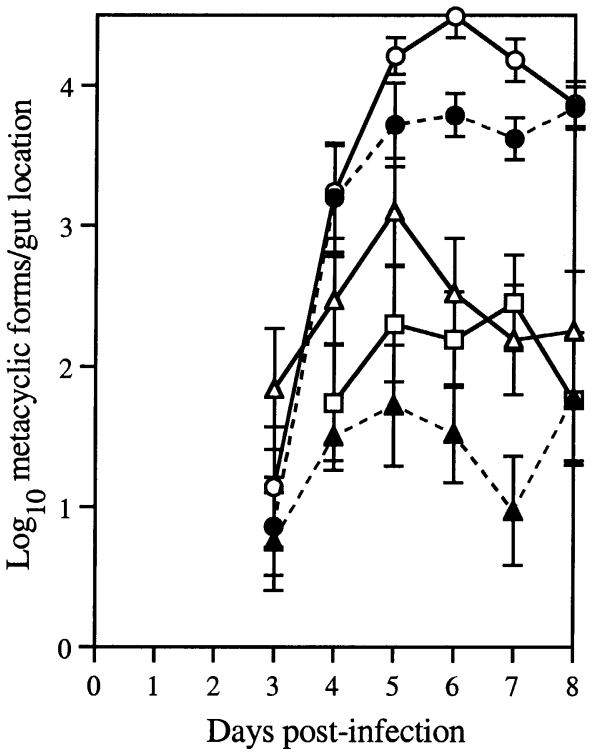

Fig. 3. The occurrence of metacyclic promastigotes of Leishmania mexicana within different gut regions of Lutzomyia longipalpis. Infected flies were dissected into foregut $(\square)$, cardia $(\bigcirc)$, thoracic midgut $(\bigcirc)$, anterior abdominal midgut $(\triangle)$, and posterior abdominal midgut $(\boldsymbol{\Delta})$. The number of metacyclic promastigotes in each gut region during the course of infection is shown. Each point represents the geometric mean of at least 10

flies \pm standard error. These data were collected from the same group of flies as in Fig. 4.

against this background. Initially the parasites are confined to the bloodmeal encased in the peritrophic matrix, and use the bloodmeal as their main source of nutrients (bloodmeal phase). When bloodmeal digestion is completed the sandflies feed on sugar meals and the parasites migrate forwards. In the current study the digestion of the bloodmeal was monitored by determining the haemoglobin content of flies and by recording the day on which defaecation of bloodmeal remnants first occurred (data not shown). Haemoglobin content of infected flies decreased rapidly over the first 3 days, indicating that this was the main period when bloodmeal digestion was occurring. Measurements remained stable thereafter at background levels. Defaecation of bloodmeal remnants for the first time was most frequent on day 2 , followed by day 3 , as flies removed the waste products of digestion. These data indicate that the bloodmeal phase of development lasted for the first 3 days, and was then superceded by the sugarmeal phase. The parasite morphological form that negotiates this transition appears to be the nectomonad promastigote, which reached a sharp peak in prevalence on day 3 (Fig. 1B).

Development of Leishmania mexicana in different gut regions

The data reported thus far relate to the overall pattern of development, taking the sandfly gut as a whole. However, infections are not spatially uniform. To understand the distribution of parasites within the gut with respect to time, a further series of infections was performed in which guts from infected flies were dissected and subdivided into separate regions. The total number of parasites in the posterior abdominal midgut, anterior abdominal midgut, thoracic midgut, cardia region and foregut were determined over an 8-day period (Fig. 2A). The overall pattern of these infections was similar to that shown in Fig. 1A, peaking on day 6 at 70000 parasites/fly. For the first 2 days the infections were largely confined to the abdominal midgut (within the peritrophic matrix), only a very small number of promastigotes reaching the thoracic midgut on day 2 (average less than 10/fly). Over these first 2 days the number of parasites increased rapidly in the abdominal midgut, especially in the anterior portion, day 2 representing the peak number in the abdominal midgut. On day 3 parasites increased to substantial numbers in the thoracic midgut and first appeared in the cardia region, and these 2 regions contained the majority of the parasite population from day 4 onwards. Thus, expansion of the population from day 3 forward was accompanied by anterior migration. Noticeable gel-like material, the developing PSG plug, was first observed in the thoracic midgut and cardia regions from day 4 onwards. On day 4 parasites were also first detected in the foregut. These never reached high numbers, but remained at approximately the same level thereafter, varying between 100 and 400 parasites/fly.

In each gut region the morphology of the parasites was also assessed. The data for the posterior and anterior abdominal midgut (Fig. 2B, C) showed a broadly similar distribution of forms with time. One interesting difference was the prevalence of procyclic and nectomonad promastigotes in the abdominal midgut on day 3. Nectomonad promastigotes were relatively concentrated in the anterior part of the abdominal midgut $(65 \%$ vs $46 \%$ ), compared with procyclic promastigotes that were relatively concentrated in the posterior abdominal midgut $(30 \%$ vs $10 \%$ ). Thus nectomonad promastigotes that have developed from procyclic promastigotes accumulate in an anterior position in the abdominal midgut, a position that favours their role in spreading the infection forwards to the thoracic midgut. This interpretation is supported by analysis of the thoracic midgut population (Fig. 2D). The small number of parasites that were found here on day 2 were mainly nectomonad forms $(80 \%)$. They remained the major form on day $3(64 \%)$ when there was a substantial increase in the thoracic midgut population (Fig. 2A), indicating that nectomonad promastigotes were the main form responsible for anterior progression of the infection. Subsequently these were replaced in the main by leptomonad promastigotes, and lower numbers of haptomonad and metacyclic forms. 
A.

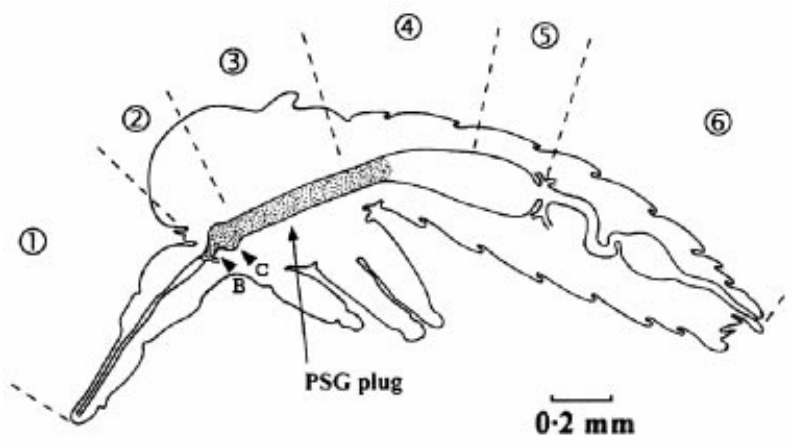

B.

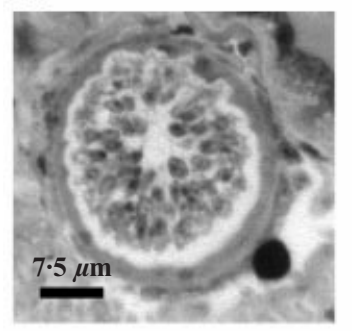

C.

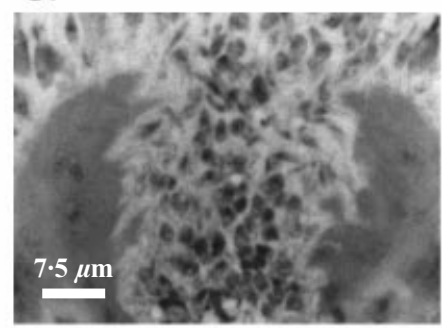

D.

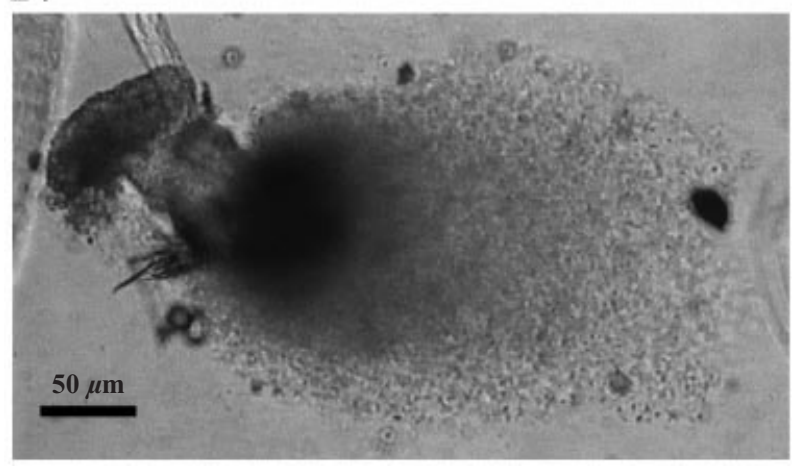

Fig. 4. Isolation of the PSG plug from the gut of day 8 Leishmania mexicana-infected Lutzomyia longipalpis. (A) Saggital section through a female sandfly showing distension of the thoracic midgut and typical position of the sausage-shaped PSG plug (redrawn after Jobling, 1987). The diagram shows the gut subdivided into: 1 , foregut; 2 , cardia; 3 , thoracic midgut; 4 , anterior abdominal midgut; 5 , posterior abdominal midgut; and 6 , hindgut. The cardia region spans the very anterior part of the thoracic midgut, the stomodeal valve and the oesophagus. The arrowheads labelled $\mathrm{B}$ and $\mathrm{C}$ indicate the positions of the histological sections described below. (B) Transverse section through the oesophagus, which is occluded by attached haptomonad promastigotes and parasites embedded in PSG. (C) Longitudinal section through the stomodeal valve, which is distended and held open by parasites embedded in PSG. (D) Appearance of an intact PSG plug dissected from the thoracic midgut, viewed by phase contrast microscopy of live material. Remnants of the stomodeal valve can be seen still attached (left).
Leptomonad forms became the dominant form in the thoracic midgut from day 4 onwards, coincident with the formation of the PSG plug. A similar pattern of colonization was observed in the cardia region (Fig. 2E). The colonizing forms were again mainly nectomonad promastigotes, which were then replaced by leptomonad promastigotes. One significant difference was that as the infections progressed the cardia population came to be dominated by metacyclic promastigotes, approximately $60 \%$ from days $6-8$, in comparison with the thoracic midgut immediately behind ( $42 \%$ on day 8$)$. This effect was even more pronounced in the final region of the gut examined, the foregut (Fig. 2F). Parasites were first noted in this location on day 4 , and the major forms present were metacyclic promastigotes $(56 \%)$ and leptomonad promastigotes $(38 \%)$. By day 6 the prevalence of metacyclic promastigotes had risen to $74 \%$ in the foregut, the highest proportion found in any gut location. The foregut was the only gut location in which paramastigotes were observed, but these did not exceed a prevalence of $4 \%$.

\section{Metacyclic promastigotes in the cardia region}

The ability of sandflies to transmit metacyclic promastigotes will depend on both the numbers of these forms present in the flies at a given time-point, and the location of these within the gut. To address these questions, the data shown in Fig. 2 were used to derive the numbers found in different gut locations with time (Fig. 3). Low numbers $(<100$ per location) of metacyclic forms were observed as early as day 3 in most locations, although they were not present in the foregut until day 4. As noted above, the numbers found in the foregut were always relatively low, the maximum being an average of 281 on day 7. These promastigotes are in the best position for transmission, and would certainly be expected to contribute to the infective dose. The few metacyclic promastigotes found in the anterior and posterior abdominal midgut (maximum numbers 1250 and $60 /$ fly, respectively) are unlikely to play a significant role in transmission by virtue of their position. The largest population was found in the cardia, which reached a maximum of 31070 metacyclic promastigotes, followed by the thoracic midgut (maximum 6950). The cardia population is located next to the foregut, at the anterior tip of the PSG plug that extends backwards, blocking the thoracic midgut. Therefore, a significant source of metacyclic promastigotes for transmission is likely to be the cardia population.

\section{Analysis of the PSG plug parasite population}

The PSG plug found in the sandfly gut has been proposed to play a role in the transmission of Leishmania, but the precise location of this material and its cellular content have not been analysed 
Table 2. Effect of infection on the size of the cardia (stomodeal valve) and thoracic midgut

(Diameters were estimated from measurements of 10 normal and 10 infected dissected guts. Cross-sectional area and volumes were derived assuming that the gut regions are cylindrical in shape. ${ }^{*}$ Fold $\uparrow$ is the ratio of infected: uninfected measurements, showing the increase resulting from infection.)

\begin{tabular}{|c|c|c|c|c|c|c|}
\hline Region & & $\begin{array}{l}\text { Diameter } \\
(\mu \mathrm{m})\end{array}$ & *Fold $\uparrow$ & $\begin{array}{l}\text { Cross-sectional } \\
\text { area }\left(\mu \mathrm{m}^{2}\right)\end{array}$ & Volume (nl) & Fold $\uparrow$ \\
\hline \multirow{2}{*}{ Cardia } & Normal & $81 \cdot 25$ & \multirow{2}{*}{$1 \cdot 5$} & 5185 & $0 \cdot 23$ & \multirow{2}{*}{$2 \cdot 3$} \\
\hline & Infected & $123 \cdot 75$ & & 12028 & 0.54 & \\
\hline \multirow{2}{*}{ Thoracic midgut } & Normal & 58.75 & \multirow{2}{*}{$1 \cdot 7$} & 2711 & $1 \cdot 08$ & \multirow{2}{*}{$2 \cdot 9$} \\
\hline & Infected & $100 \cdot 0$ & & 7854 & $3 \cdot 14$ & \\
\hline
\end{tabular}

previously. On dissection, noticeable gel-like material could be observed in the thoracic midgut/ cardia region from day 4 onwards, increasing in amount as the infection reached its peak. Infected flies retained a PSG plug for the duration of their life. In uninfected flies the stomodeal valve is usually shut, and therefore presents a barrier to transmission. However, in the cardia region parasites embedded in the plug were seen to extend through the stomodeal valve, which was occluded and held open, and into the oesophagus (Fig. 4A-C). Posteriorly, the PSG plug extended back from the cardia through the thoracic midgut and, in some flies, into the abdominal midgut. Intact infected dissected guts were noticeably distended in the thoracic midgut/ cardia region compared to those from uninfected flies, and the extent of this distension was measured (Table 2). The thoracic midgut was approximately 3 times its normal volume in infected flies. These measurements demonstrate the profound effect that the presence of the leishmanial infection has on the sandfly gut, and indicate that the PSG plug exerts considerable mechanical pressure on the surrounding gut wall. Careful dissection of infected flies enabled the PSG plug to be removed intact from the remaining contents of the gut. When isolated the PSG plug had a sausage-like appearance that expanded in size upon release from the confines of the thoracic midgut (Fig. 4D). The parasites embedded in the centre of the plug often appeared immobile in live preparations, but could swim away if the plug was gently disrupted in culture medium, indicating that they remained viable. Parasites at the periphery of the plug were highly motile, could detach themselves, and were predominantly metacyclic promastigotes. The morphological forms present in the PSG plug and in the remainder of the gut were analysed and those found within the plug, PSG plug forms, were significantly different in proportion to those found elsewhere, the remaining midgut forms (Fig. 5A, B). The dominant form within the PSG plug was the leptomonad promastigote accounting for $53 \%$ of the total, whilst elsewhere metacyclic $(49 \%)$ and leptomonad $(39 \%)$ promasti- gotes were the most prevalent. Although a relatively minor population, the PSG plug population contained the highest proportion of haptomonad forms with expanded flagellar tips $(10 \%)$. In day 8 infections the number of parasites in the plug averaged 15500 (range 1200 to 44000 ), and these represented on average $80 \%$ of the total population in these mature infections (range 54 to $100 \%$ ). The number in the rest of the gut averaged 1400/sandfly (range 600 to 22800 ).

Interestingly, the majority $(\sim 75 \%)$ of metacyclic promastigotes present in these flies with mature infections were associated with the PSG plug, rather than being in the rest of the midgut. To investigate this observation further the PSG plugs isolated from another series of sandflies were subdivided into 3 portions of equal size: the anterior pole (including the cardia population), mid-section and posterior pole. Fig. 5C-E shows the morphological analysis of these subsections of the PSG plugs. An average of $40 \%$ of the parasites were in the anterior poles (10000), $44 \%$ in the mid-sections (12000), and 16\% in the posterior poles (3800). Thus the parasites are concentrated towards the anterior end of the PSG plug. The developmental forms found in the two poles were similar to each other, but different to the population found in the mid-section. The latter was strongly dominated by leptomonad promastigotes $(58 \%)$, whereas at the poles there were roughly equal proportions of leptomonad forms and metacyclic promastigotes. Thus the pole populations are intermediate between those in the mid-section of the PSG plug and those parasites found elsewhere in the gut. These data indicate that metacyclic promastigotes differentiate from leptomonad promastigotes at the anterior and posterior poles of the PSG plug. However, numerically a clear gradient existed, with $58 \%$ of the PSG plug metacyclic forms occurring at the anterior pole (cardia region), $23 \%$ in the midsection, and $19 \%$ in the posterior pole.

Most experimental work on Leishmania is conducted using in vitro cultures, in which densities are normally maintained in the range $5 \times 10^{5}$ to $5 \times 10^{7}$ parasites $/ \mathrm{ml}$. In contrast, observation of the PSG 

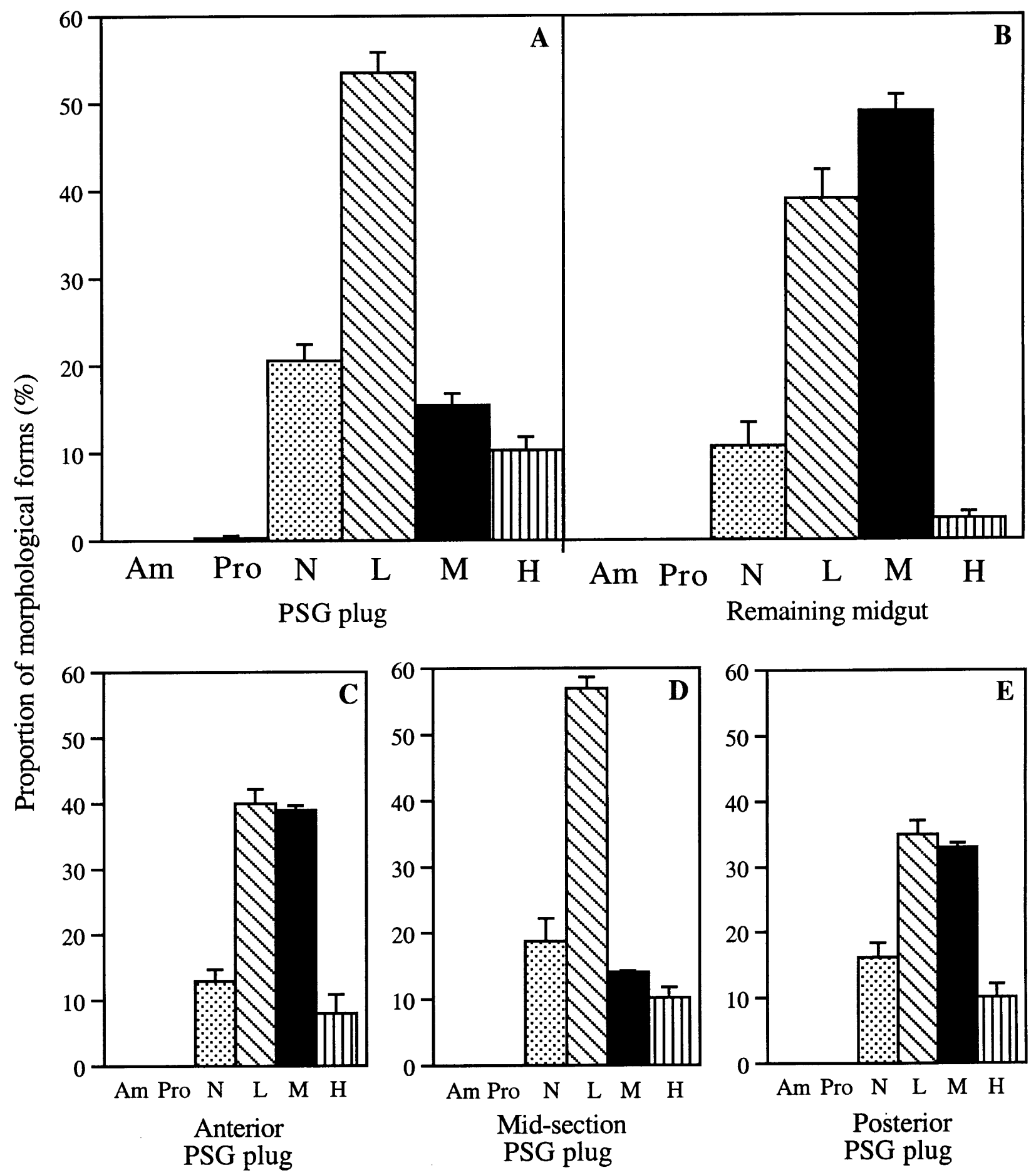

\section{Morphological forms}

Fig. 5. The developmental profile of Leishmania mexicana associated with the PSG plug. Guts from flies with 8-dayold infections were dissected and the gel-like plug removed: (A) the separated PSG plug; and (B) remaining gut samples, were assessed for parasite morphology. The proportions of amastigotes (Am), procyclic promastigotes (Pro), nectomonad promastigotes $(\mathrm{N})$, leptomonad promastigotes $(\mathrm{L})$, metacyclic promastigotes $(\mathrm{M})$ and haptomonad promastigotes $(\mathrm{H})$ are shown. Each bar represents the arithmetic mean \pm standard error of 20 infected flies, from the combination of 2 independent experiments. Further preparations were analysed for distribution within the plug: (C) anterior pole; (D) mid-section; and (E) posterior pole. Each bar represents the arithmetic mean \pm standard error of 10 infected flies.

plug, revealed a mass of parasites at very high density. Knowledge of the numbers of parasites per fly, their location in the gut, and calculation of the volume of the sandfly gut (Table 2), enabled an estimate of the density achieved in vivo to be made. At its peak, the total number of parasites per fly was 
Table 3. Feeding activity compared to infection levels in sandflies taking a second meal of medium by membrane feeding

(Combined data from 3 independent experiments.)

\begin{tabular}{lllll}
\hline \hline Type of feed & \% with no infection & $\%$ with light infections & \% with heavy infections & $\begin{array}{l}\text { Average midgut } \\
\text { infection level }\end{array}$ \\
\hline All flies $(n=60)$ & 30 & 13 & 57 & $4 \cdot 40 \times 10^{4}$ \\
Probe $(n=20)$ & 35 & 10 & 55 & $4 \cdot 04 \times 10^{4}$ \\
Full $(n=15)$ & 73 & 13 & 13 & $2 \cdot 26 \times 10^{4}$ \\
Partial $(n=25)$ & 0 & 16 & 84 & $5 \cdot 02 \times 10^{4}$ \\
\hline \hline
\end{tabular}

Table 4. Feeding activity compared to infection levels in sandflies taking a second meal of blood and mice (Combined data from 2 independent experiments.)

\begin{tabular}{lccc}
\hline \hline Type of feed & \% with no infection & \% with light infections & \% with heavy infections \\
\hline All flies $(n=176)$ & 24 & 20 & 57 \\
None/probe $(n=87)$ & 23 & 21 & 56 \\
Full $(n=42)$ & 48 & 33 & 19 \\
Partial $(n=47)$ & 4 & 9 & 87 \\
\hline \hline
\end{tabular}

in the range 35000 to 70000 , these being confined mainly to the thoracic midgut and cardia regions. We estimate that the volume of these regions of the gut (allowing for distention) was approximately $4 \mathrm{nl}$ and, therefore, that the density on day 6 was $1-2 \times 10^{10} / \mathrm{ml}$. This compares with an initial density of $\sim 4 \times 10^{6}$ amastigotes $/ \mathrm{ml}$ in the fresh bloodmeal. Therefore, although the overall increase in numbers per fly was relatively modest (15 to 30 -fold), in mature infections the parasites achieved extremely high densities (2500 to 5000 -fold increase) through being confined in a small area, densities far greater than can be obtained by in vitro culture.

\section{Effect of presence and size of an infection on the feeding success of sandflies}

The PSG plug has been proposed to assist transmission, the 'blocked-fly hypothesis' (Stierhof et al. 1999). One prediction of this hypothesis is that the most heavily infected flies with the largest PSG plugs should find the greatest difficulty in taking a bloodmeal. In persisting in their attempts to feed such flies would be the most effective in transmission of leishmaniasis. This prediction was tested in the current study by examination of the feeding activity of Lu. longipalpis infected with L. mexicana. In the first series of experiments, infected sandflies were allowed to feed through a chick skin membrane. After a single feeding attempt, each fly was then dissected and the gut examined to determine whether a full meal had been taken (midgut fully distended), a partial meal only, or no meal at all. Since each fly was removed immediately after having withdrawn its proboscis from the chick skin feeder, those with no meal were known to be flies that probed, but did not feed. Each gut was then homogenized and the number of parasites per fly determined by haemocytometer counting (Table 3). The distribution of infections in those flies that showed any feeding activity $(30 \%$ uninfected, $13 \%$ light infections, $57 \%$ heavy infections), and their average infection intensity (44000/infected fly) were the same as those in the general population of flies used in these experiments. Similarly, infection levels in the subpopulation of flies that probed but which did not subsequently feed were also very similar to the total population. These data indicate that flies that attempted to feed were a random sample of the total population, and that the presence of an infection in a sandfly did not appear to influence its desire to attempt to feed or to insert its proboscis into the feeder. In contrast, in sandflies that inserted their probosces and went on to take a full or partial meal, the presence of an infection had a dramatic effect on the outcome. Of those flies that took a full meal, $73 \%$ were found to be uninfected upon dissection, a much higher proportion than in the overall population $(30 \%)$, whereas only $13 \%$ had heavy infections. Thus the presence of an infection had a strong negative effect on the ability of flies to take a full meal. Conversely, of the flies that could only take a partial meal, $84 \%$ of these were heavily infected, and none were uninfected.

A second set of experiments was performed in which infected sandflies were allowed to feed on anaesthetized BALB/c mice. This allowed a larger set of data to be collected and in an experimental situation more akin to natural transmission. After a 30 min exposure, the mice were removed from the cage, and all the flies were then dissected to establish their feeding status and infection level (Table 4). In 
the total population of flies $24 \%$ were uninfected, $20 \%$ had light infections and $57 \%$ were heavily infected. As above, flies without any bloodmeal displayed a very similar pattern of infections. These represent a combination of flies that did not attempt to feed and those that inserted their probosces but did not ingest any blood. Flies that did take a bloodmeal again showed very different infection patterns. As with the membrane feeding experiments described above, flies with a full bloodmeal contained a higher proportion without infection ( $48 \%$ vs $24 \%$ ), whereas those with partial bloodmeals contained a higher proportion of heavily infected flies $(87 \%$ vs $57 \%$ ). All of the mice exposed to the bites of infected sandflies went on to develop multiple, rapidly growing progressive cutaneous lesions. Collectively, these 2 sets of data demonstrated that once a fly began feeding, the presence of an existing $L$. mexicana infection had a strong influence on the outcome of that feeding attempt. Heavily infected flies had more difficulty in taking a full meal than flies with lighter infections or uninfected flies.

\section{DISCUSSION}

This study is the first to quantitate Leishmania mexicana development in vivo, with respect to both time and gut location. Consequently, we provide a new understanding of the different morphological stages of Leishmania found in the sandfly and their individual functions. We also propose the creation of a new life-cycle stage, the leptomonad promastigote.

Previously, the development of L. mexicana has been studied in both natural (Lutzomyia diabolica) and experimental hosts (Lu. shannoni, Lu. abonnenci) (Lawyer et al. 1987; Walters et al. 1987). The results of these previous studies are in agreement with the more quantitative analysis provided in the current report. Amastigotes transformed to procyclic promastigotes, a form confined to the environment of the bloodmeal in the abdominal midgut. Amastigote to promastigote transformation was accompanied by some mortality, a phenomenon that has also been reported previously in L. major/Phlebotomus papatasi (Pimenta et al. 1997). This was ascribed to the change in surface glycocalyx that accompanies transformation, making the parasite temporarily vulnerable to trypsin attack. Consequently, Leishmania establishment in the sandfly starts from a reduced population of transformed procyclic promastigotes. The main function of the procyclic promastigote is to replicate within the nutritionally rich environment of the bloodmeal and establish the initial infection. As the bloodmeal phase of development nears its end, after 2-3 days of infection, procyclic promastigotes transformed into nectomonad promastigotes. These accumulated in the anterior abdominal midgut, also noted previously (Walters et al. 1989b), and were responsible for anterior spread of the infection towards the thoracic midgut and cardia region.

Nectomonad promastigotes began to escape the peritrophic matrix-encased bloodmeal before digestion was completed, as early as day 2, supporting a role for these forms in establishment of the infection outside the bloodmeal. The escape of nectomonad promastigotes may be assisted by the secretion of a parasite chitinase (Schlein, Jacobson \& Messer, 1992; Pimenta et al. 1997), or during a window of opportunity when sufficient autolysis of the PM had occurred to allow a minority of correctly positioned parasites through, or a combination of the two. Nectomonad promastigotes were the first forms to arrive and colonize the anterior midgut, and were the precursors of leptomonad promastigotes. Two important functions can be ascribed to leptomonad promastigotes that justify the creation of a separate category (a), production of the PSG plug, i.e. the 'blocked fly' and (b), as developmental precursors of metacyclic promastigotes. In generating the blocked fly the leptomonad promastigotes are assisted by haptomonad forms, which anchor themselves to the chitinous surface of the stomodeal valve via hemidesmosome-like plaques at the ends of their flagella (Lawyer et al. 1987; Killick-Kendrick et al. 1974; Molyneux \& Killick-Kendrick, 1987). Since these are a small population their developmental origin is difficult to determine, but their size suggests an origin from leptomonad promastigotes.

The number and distribution of metacyclic promastigotes indicated that the major site of metacyclogenesis was at the anterior pole of the PSG plug, in a position suitable for transmission. Leptomonad promastigotes trapped within the gel-like matrix differentiated into metacyclic promastigotes, which then accumulated in the cardia region to provide the source of infective forms. This conclusion is supported by the results of Lawyer et al. (1990), who severed the gut at the oesophagus of $L$. major-infected Phlebotomus duboscqi and expressed the contents. Spewing from the cardia was a nearhomogenous population of metacyclic promastigotes, which was followed, with the application of pressure, by a mixture of forms in association with gel-like material (i.e. PSG). The motility of metacyclic promastigotes was apparently not hindered by PSG. This latter observation may explain the ability of metacyclic promastigotes to concentrate at the anterior of the PSG obstruction, and explain the remarkable homogeneity of parasites egested from the mouthparts when L. major-infected P. papatasi were capillary fed (Saraiva et al. 1995).

The high density of anterior midgut infections would promote the accumulation of high concentrations of secreted filamentous proteophosphoglycans (fPPG) that are known to have gel-forming properties (Stierhof et al. 1999; Ilg et al. 1996). Consequently, 2 features of PSG are likely to 
stimulate metacyclogenesis. First, against the background of a weakly acidic anterior midgut in $L u$. longipalpis ('Tang, Anez \& Bates, 1998), the fPPG component is a strongly negatively charged acidic molecule, and a low $\mathrm{pH}$ is known to stimulate metacyclogenesis in vitro (Bates \& Tetley, 1993; Zakai, Chance \& Bates, 1998). Secondly, the gelatinous nature of PSG coupled with the high cell density is likely to cause local depletion of oxygen, and anaerobiosis is also known to stimulate metacyclogenesis in vitro (Mendez et al. 1999). Additionally, after differentiation from leptomonad promastigotes in the middle of the PSG plug metacyclic promastigotes may migrate to either pole, concentrating at the anterior in response to a chemotactic cue. Recently, a variety of sugars were demonstrated to have a positive chemotactic effect on L. amazonensis and L. chagasi stationary-phase promastigotes in vitro (Oliveira, Maria \& Gontijo, 2000). The possibility that Leishmania respond to sugars or saliva released from the crop that could form a gradient within the sandfly midgut remains to be addressed.

In the current study, production of the PSG plug was first observed at the stomodeal valve (cardia region), which then expanded bidirectionally. The resulting swelling of the thoracic midgut and cardia detailed in the current report has also been noted before (Lawyer et al. 1987). Damage and dysfunction of the stomodeal valve has been implicated in the transmission of leishmaniasis, resulting from the action of chitinases secreted by attached promastigotes (Schlein et al. 1992). In contrast, we found no evidence of damage to this structure in L. mexicanainfected Lu. longipalpis or in the natural parasite/ vector combination of $L$. chagasi/Lu. longipalpis (A. Y. Ismaeel, unpublished results). Instead, it was observed that pressure from the expanding PSG plug forced the valve open and this may impair its function, facilitating a reflux of blood and parasites to enter the bite site. Furthermore, the opening of the stomodeal valve is presumably essential for expansion of the infection into the foregut.

The mechanical properties of PSG are such that it has been proposed to play a significant role in the transmission of leishmaniasis (Stierhof et al. 1999). It has been frequently noted that Leishmaniainfected sandflies experience difficulty in taking a full engorgement of blood and yet are still able to transmit the parasite (Killick-Kendrick, 1979; Beach et al. 1984; Beach, Kiilu \& Leeuwenburg, 1985; Jefferies, Livesey \& Molyneux, 1986). In this study, clear evidence is given for a relationship between infection intensity and the size of the bloodmeal obtained, using sample sizes that for the first time allow definite conclusions to be drawn. Whether feeding on a mouse or membrane feeding system, flies with no infections fully engorged on blood with ease, whereas most flies with heavy infections could only take partial meals at best. Interestingly, the presence of an infection and therefore the PSG plug did not influence the fly's desire to feed, only its ability. Although these data lend considerable support to the 'blocked fly hypothesis' and the role of PSG, the relationship between the size of the PSG blockage and the transmission potential of the fly merits further investigation.

In summary, the results of this study provide significant new insights into the development of Leishmania in its vector, and will prove useful to the interpretation of mutant Leishmania phenotypes expressed in sandflies. In particular, the role of the PSG plug in adapting the infected sandfly for transmission is established through alteration of the feeding behaviour of the fly and conditioning the anterior midgut for efficient metacyclogenesis. This study is part of our ongoing investigations into various aspects of Leishmania transmission.

M.E.R. was supported by a Medical Research Council Studentship. This investigation also received financial support from the UNDP/World Bank/WHO Special Programme for Research and Training in Tropical Diseases (TDR). The technical assistance of Davina Moor in maintenance of sandflies is gratefully acknowledged.

\section{REFERENCES}

BATES, P. A. (1997). Infection of phlebotomine sandflies with Leishmania. In The Molecular Biology of Insect Disease Vectors (ed. Crampton, J. M., Beard, C. B. \& Louis, C.), pp. 112-120. Chapman \& Hall, London. BATES, P. A. \& TETLEY, L. (1993). Leishmania mexicana: induction of metacyclogenesis by cultivation of promastigotes at acidic pH. Experimental Parasitology 76, 412-423.

BEACH, R., KIILU, G., HENDRICKS, L., OSTER, C. \& LEEUWENBERG, J. (1984). Leishmania major in Kenya: transmission to a human by the bite of a naturally infected Phlebotomus duboscqi sandfly. Transactions of the Royal Society of Tropical Medicine and Hygiene 78, 747-751.

BEACH, R., KIILU, G. \& LEEUWENBURG, J. (1985). Modification of sandfly biting behavior by Leishmania leads to increased parasite transmission. American Fournal of Tropical Medicine and Hygiene 34, 278-282. BRIEGEL, H., LEA, A. O. \& KLOWDEN, M. J. (1979).

Hemoglogbinometry as a method for measuring bloodmeal sizes of mosquitoes (Diptera: Culicidae). Fournal of Medical and Veterinary Entomology 15, 235-238.

ILG, T., STIERHOF, Y.-D., CRAIK, D., SIMPSON, R., HANDMAn, E. \& BACIC, A. (1996). Purification and structural characterization of a filamentous mucin-like proteophosphoglycan secreted by Leishmania parasites. Fournal of Biological Chemistry 271, 21583-21596.

ismaeel, A. Y., GaRmSON, J. C., MOLYNEUX, D. H. \& BATES, P. A. (1998). Transformation, development, and transmission of axenically cultured amastigotes of Leishmania mexicana in vitro and in Lutzomyia longipalpis. American Fournal of Tropical Medicine and Hygiene 59, 421-425. 
JEFFERIES, D., LIVESEY, J. L. \& MOLYNEUX, D. H. (1986). Fluid mechanics of bloodmeal uptake by Leishmaniainfected sandflies. Acta Tropica 43, 43-53.

jobling, в. (1987). Anatomical Drawings of Biting Flies. Dorset Press, Dorchester.

KILLICK-KENDRICK, R. (1979). Biology of Leishmania in phlebotomine sandflies. In Biology of the Kinetoplastida, Vol. 2, (ed. Lumsden, W. H. R. \& Evans, D. A.), pp. 395-460. Academic Press, London/New York.

KILLICK-KENDRICK, R. (1990). The life-cycle of Leishmania in the sandfly with special reference to the form infective to the vertebrate host. Annales de Parasitologie Humaine et Comparée 65 (Suppl. 1), $37-42$.

KILLICK-KENDRICK, R., MOLYNEUX, D. H. \& ASHFORD, R. W. (1974). Leishmania in phlebotomid sandflies 1. Modifications of the flagellum associated with attachment to the mid-gut and oesophageal valve of the sandfly. Proceedings of the Royal Society of London, B 187, 409-419.

LAWYER, P. G., NGUMBi, P. M., ANJILI, C. O., ODONGO, S. O., Mebrahtu, Y. B., Githure, J. I., KOECH, D. K. \& RoBerts, C. R. (1990). Development of Leishmania major in Phlebotomus duboscqi and Sergentomyia schwetzi (Diptera: Psychodidae). American Fournal of Tropical Medicine and Hygiene 43, 31-43.

LAWYER, P. G., YOUNG, D. G., BUTLER, J. F. \& AKIN, D. E. (1987). Development of Leishmania mexicana in Lutzomyia diabolica and Lutzomyia shannoni (Diptera: Psychodidae). Fournal of Medical Entomology 24, 347-355.

MENDEZ, S., FERNANDEZ-PEREZ, F. J., DE LA FUENTE, C., CUQUERELla, M., GOMEZ-MUNOZ, M. T. \& ALUNDA, J. M. (1999). Partial anaerobiosis induces infectivity of Leishmania infantum promastigotes. Parasitology Research 85, 507-509.

MODI, G. B. (1997). Care and maintenance of phlebotomine sandfly colonies. In The Molecular Biology of Insect Disease Vectors (ed. Crampton, J. M., Beard, C. B. \& Louis, C.), pp. 21-30. Chapman \& Hall, London.

MOLYNEUX, D. H. \& KILLICK-KENDRICK, R. (1987). Morphology, ultrastructure and life cycles. In The Leishmaniases, Vol. 1 (ed. Killick-Kendrick, R. \& Peters, W.), pp. 121-176. Academic Press, London. oliveira, J. S., Maria, M. N. \& Gontijo, N. F. (2000). A sensitive method for assaying chemotaxic responses of Leishmania promastigotes. Experimental Parasitology 96, 187-189.

PIMENTA, P. F. P., MODI, G. B., PEREIRA, S. T., Shahabuddin, M. \& SACKs, D. L. (1997). A novel role for the peritrophic matrix in protecting Leishmania from the hydrolytic activities of the sandfly midgut. Parasitology 115, 359-369.

Pimenta, P. F. P., TURCO, S. J., McCONVILle, M. J., LAWYER, P. G., PERKINS, P. V. \& SACKS, D. L. (1992). Stage-specific adhesion of Leishmania promastigotes to the sandfly midgut. Science 256, 1812-1815.
SACKS, D. L. (1989). Metacyclogenesis in Leishmania promastigotes. Experimental Parasitology 89, 101-103.

SACKs, D. L., SARaiva, E. M., ROWTON, E., TURCO, S. J. \& Pimenta, P. F. (1994). The role of the lipophosphoglycan of Leishmania in vector competence. Parasitology 108 (Suppl.), S55-S62.

SÁdLOVÁ, J., REISHIG, J. \& VOLF, P. (1999). Prediuresis in female sandflies (Diptera: Psychodidae). European Fournal of Entomology 95, 643-647.

SARAIVA, E. M. B., PIMENTA, P. F. P., BRODIN, T. N., Rowton, E., MODI, G. \& SACKS, D. L. (1995). Changes in lipophosphoglycan and gene expression associated with the development of Leishmania major in Phlebotomus papatasi. Parasitology 111, 275-287.

SCHLEIN, Y., JACOBSON, R. L. \& MESSER, G. (1992). Leishmania infections damage the feeding mechanism of the sandfly vector and implement parasite transmission by bite. Proceedings of the National Academy of Sciences, USA 89, 9944-9948.

ShortT, H. E. \& SWaminath, c. s. (1928). The method of feeding of Phlebotomus argentipes with relation to its bearing on the transmission of kala-azar. Indian Fournal of Medical Research 15, 827-836.

STIERHOF, Y.-D., BATES, P. A., JACOBSON, R. L., ROGERS, M. E., SCHLEIN, Y., HANDMAN, E. \& ILG, T. (1999). Filamentous proteophosphoglycan secreted by Leishmania promastigotes forms gel-like threedimensional networks that obstruct the digestive tract of infected sandfly vectors. European Fournal of Cell Biology 78, 675-689.

TANG, Y., ANEZ, N. \& Bates, P. A. (1998). Phenol red method for measuring the $\mathrm{pH}$ of the gut contents in Lutzomyia longipalpis (Psychodidae: Diptera). Chinese Fournal of Parasitology and Parasitic Diseases 16, 62-66.

WALTERS, L. L. (1993). Leishmania differentiation in natural and unnatural sandfly hosts. Fournal of Eukaryotic Microbiology 40, 196-206.

WALTERS, L. L., CHAPlin, G. L., MODI, G. B. \& TESH, R. B. (1989a). Ultrastructural biology of Leishmania (Viannia) panamensis (= Leishmania braziliensis panamensis) in Lutzomyia gomezi (Diptera: Psychodidae): a natural host-parasite association.). American Fournal of Tropical Medicine and Hygiene 41, 295-317.

WALTERS, L. L., MODi, G. B., CHAPLIN, G. L. \& TESH, R. B. $(1989 b)$. Ultrastructural development of Leishmania chagasi in its vector, Lutzomyia longipalpis (Diptera: Psychodidae). American Fournal of Tropical Medicine and Hygiene 41, 295-317.

WAlters, L. L., MODi, G. B., TeSh, R. B. \& BURRaGe, T. (1987). Host-parasite relationship of Leishmania mexicana mexicana and Lutzomyia abonnenci (Diptera: Psychodidae). American Fournal of Tropical Medicine and Hygiene 36, 294-314.

wenyon, C. M. (1926). Protozoology, Vol. 1. Baillière, Tindall \& Cox, London.

Zakai, H., Chance, M. L. \& Bates, P. A. (1998). In vitro stimulation of metacyclogenesis in Leishmania braziliensis, L. donovani, L. major and L. mexicana. Parasitology 116, 305-309. 